\title{
Development of practical methods for increasing electromagnetic compatibility in atmospheric radio sounding systems
}

\author{
Ivan Malygin ${ }^{1, *}$ and Sergey Kudinov ${ }^{1}$ \\ ${ }^{1}$ URFU, Department of Radio Electronics and Communications, 620002 Yekaterinburg, Russia
}

\begin{abstract}
During the operation of atmospheric radio sounding systems, there appears a two-sided problem of electromagnetic compatibility with other radio-electronic systems for various purposes, ground, air and space based. Out-of-band emissions from upper-air radiosonde transmitters interfere with global satellite navigation systems and cellular systems. The paper presents the analysis of methods for reducing out-of-band emissions of aerological radiosondes and proposes the use of technology of spread spectrum communication systems in aerological complexes.
\end{abstract}

\section{Introduction}

Radio sounding systems (RSS) of the atmosphere are designed to obtain the most accurate aerological information about the thermodynamic parameters of free atmosphere, such as temperature, humidity, pressure, direction and wind speed at an altitude from ground level to $35-40 \mathrm{~km}$. In the Russian Federation, there are radar and radio navigation RSS in operation [1]. Abroad there used a radio-navigation RSSs type produced by the following manufacturers such as Vaisala, Graw, Modem, Meteolabor, Sippican, etc. According to WMO data, more than 2,000 aerological radiosondes (ARS) are launched per day in various regions across the globe [2]. During the operation of the RSS, there appears a two-sided problem of electromagnetic compatibility (EMC) with other radio-electronic means (REM) for various purposes, ground, air and space based. In the development of ARS, there are taken well-known structural and technological measures to improve EMC characteristics. However, due to the minimization of cost and ARS flight mass these methods cannot be fully used.

\section{Problem statement}

Another way for EMC increasing is connected with the development of a structural scheme for ARSs constructing, for example, based on the application of broadband generation methods of emitted signals and their digital processing in the receiver of the ground-based RSS complex. There are considered main forms of the expansion of the radiation spectrum of ARS [6]:

${ }^{*}$ Corresponding author: pit_pit2@mail.ru 
Direct spread spectrum systems based on pseudo-random sequences, including code division systems (DSSS);

-Frequency hopping (slow and fast) (FSSS);

- Systems of multiple access with spread spectrum and carrier control (CSMA);

— Systems with realignment of the temporary position of the signals (Time-hopping $\mathrm{TH})$

- Systems with linear frequency modulated signals (chirp spread spectrum - CSS)

- Systems with mixed spreading methods.

The factors determining out-of-band emissions of REM need to be considered in more detail. Reducing interference caused by unwanted emissions of ARS transmitters can be implemented by the following technical solutions [5]:

1. Optimization of transmitter architecture;

2. Filtration of transmitter radiation;

3. Modulation methods of transmitter radiation;

4. Improving the linearity of the transmitter characteristics by pre-distortion signal; use of a positive direct connection; feedback use; applying modulation feedback; use of the modulation transfer technology "Polar Loop"; implementation of the Cartesian loop technology.

For further level reduction of out-of-band emissions of the ARS, the BSSs properties modulated by pulses of a rectangular and a Gaussian shape are compared by modelling in a Matlab environment, Fig. 1. It is advisable to use phase-shifted signals as spread spectrum signals (SSS) [7]. A modulating signal consisting of N pulses can be written as follows [4]:

$$
U(t)=\sum_{n=1}^{N} a_{n} \cdot u_{0}[t-(n-1)] \tau_{0}
$$

where $u_{0}(t)=1$ for $0 \leq t \leq \tau_{0}$ is a rectangular pulse.

Each $n$-th pulse has $a_{n}$ amplitude and delays from the origin by the time $(n-1) \tau_{0}$ which equals to the total duration of all previous pulses.

The base station correlator calculates the correlation integral of the form

$$
z=\int_{0}^{T} x(t) \cdot y(t) d t
$$

where $x(t)$ is the signal at the input of a receiving device and is equal to the sum of the useful signal $y(t)$ and white Gaussian noise.

The model of SS signal spectrum formed by the M-sequence with a length of 63 elements is shown in Fig. 1.

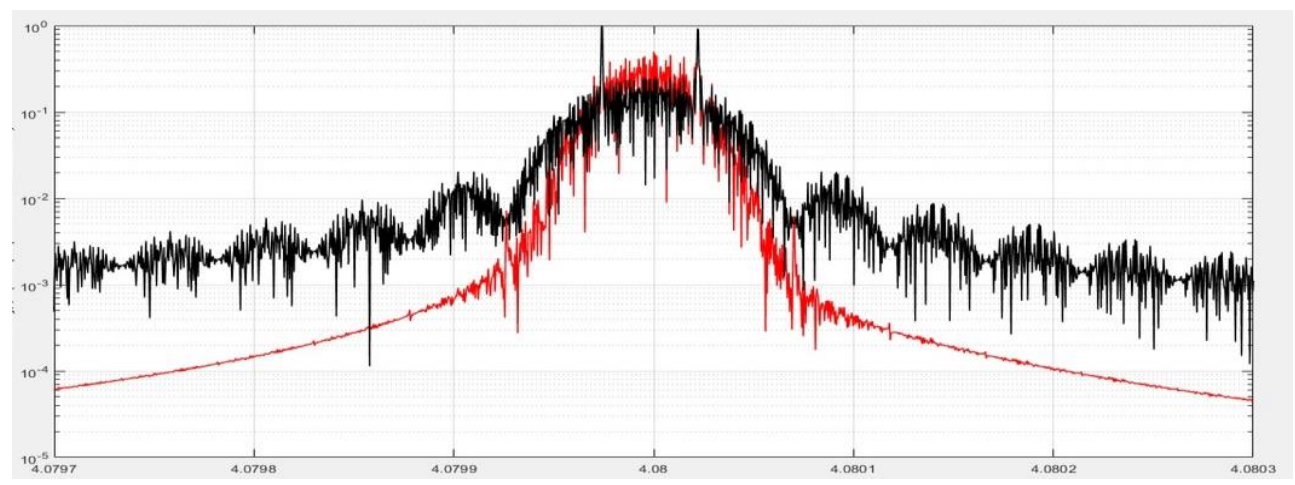

Fig. 1. Comparative image of the spectra of GFSK and FSK signals. 


\section{Development of spread spectrum equipment for a RSS}

As a result of the analysis, in order to reduce spurious emissions, methods were identified that could be implemented in RSS for optimizing the shape of modulating information pulses using Gaussian filtering, spread spectrum communication signal technology, and filtering the output radiation of the ARS transmitter with a SAW filter.

Based on the correlation reception theory of broadband phase-shifted signals, there are proposed structural schemes of the ARS transmitter and the base station receiver which are implemented in relation to RSS.

\subsection{Radiosonde transmitter design}

On the basis of conducted analysis, there was developed a block diagram of the ARP transmitting device which is shown in Fig.2.

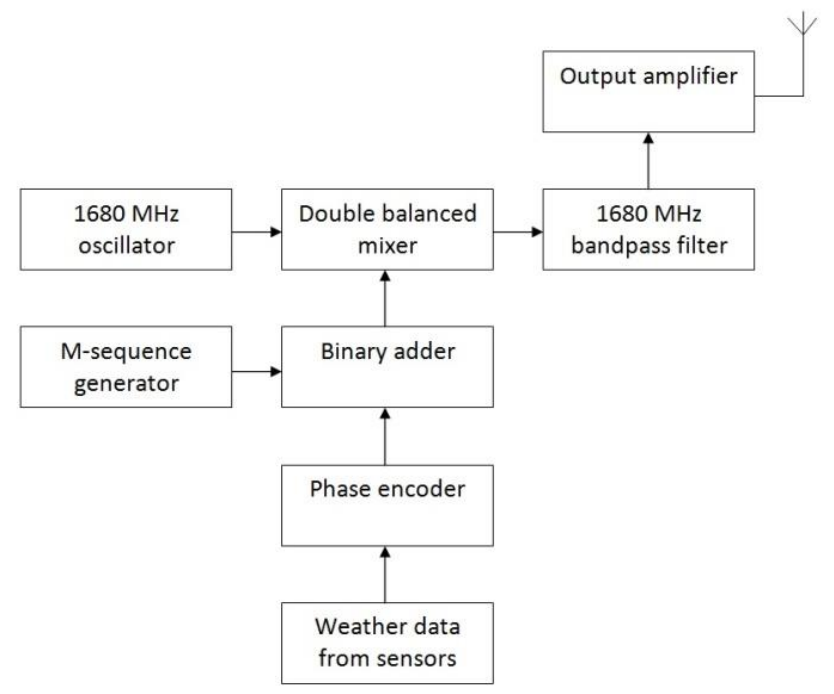

Fig. 2. Block diagram of the ARS transmitter with a spread spectrum signal.

The proposed scheme of the transmitter ARZ used the following technical solutions and components. The M-sequence generator is designed to form an M-sequence with a length of 127 elements. The modulo-2 modulator puts the information sequence and the Msequence, thereby, ensuring the spreading of the spectrum. A relative phase encoder is used to eliminate phase uncertainty in the transmission of a useful signal, since the quadrature method is not used in this case. In this case, a double balanced mixer acts as a phase modulator of a carrier signal which is formed by a master oscillator. From the output of the double balanced mixer, the modulated signal arrives at a $1680 \mathrm{MHz}$ bandpass filter. The bandpass filter $[9,10]$ provides additional suppression of out-of-band emissions relative to the signal level in the passband of $1680 \pm 10 \mathrm{MHz}$ by minus $40 \mathrm{~dB}$. In this case, the spectral density of out-of-band emissions of the PSS is lower than minus $120 \mathrm{dBW} / \mathrm{Hz}$.

\subsection{Development of a receiving device of spread spectrum signals}

The diagram of the developed receiver in canonical form is shown in Fig.3. The radio frequency part of the circuit consists of the microwave amplifier, the first mixer, the first local oscillator, the first IF filter and the first IF amplifier. 


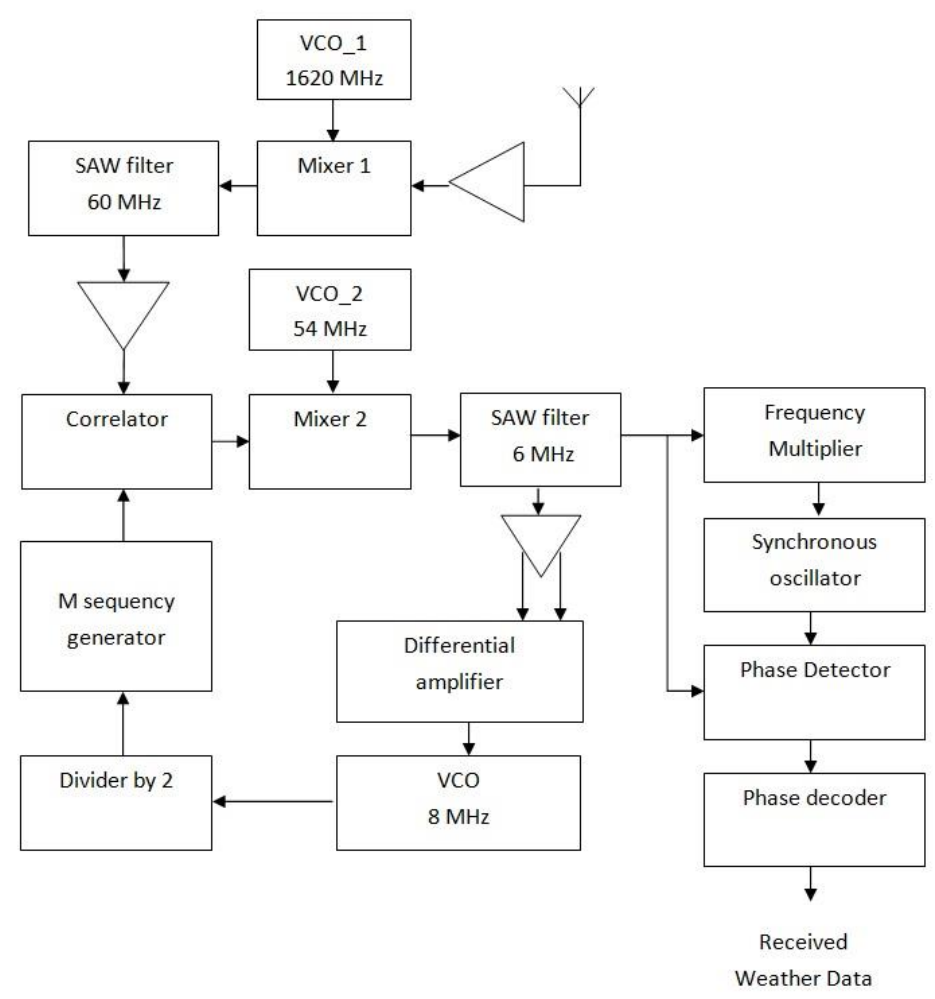

Fig. 3. Block diagram of the receiving device radar spread spectrum signal.

It is necessary to clarify some features of processing a received signal. The threechannel correlator is built according to a scheme with a various delay of the reference signal or a "sliding" reference signal [7]. In the process of searching for the synchronization mode, the pulse repetition rate of the code sequence in the receiving device changes slightly in comparison with the clock frequency sequence in the transmitter.

To achieve synchronization one of the sequences slides past the other one until they coincide. The main advantage of this correlation method is its simplicity. Time constraints are considered the key drawbacks, especially when there is a high degree of uncertainty about the phase delay between the sequences.

As noted in [7], in the receiving system with $1 \mathrm{kHz}$ frequency band of the processing device at the output of the correlator the recognition time of the synchronization mode is approximately $35 \mathrm{~ms}$.

\section{Conclusion}

1. There is a problem of radiation electromagnetic compatibility of radiosonde transmitters with REM for various purposes during the operation of aerological radiosonde systems.

2. There were considered possible methods to increase electromagnetic compatibility: optimization of the shape of modulating information pulses using Gaussian filtering, application of spread spectrum communication channel technology, filtering of the output radiation of the ARS transmitter with a SAW filter.

3. The use of proposed methods allows reducing the level of out-of-band emissions of ARS transmitters to less than minus $120 \mathrm{dBW} / \mathrm{Hz}$. 


\section{References}

1. A. Mecke, I. Lee, J.R. Baker jr., M.M. Banaszak Holl, B.G. Orr, Eur. Phys. J. E 14, 7 (2004)

2. M. Ben Rabha, M.F. Boujmil, M. Saadoun, B. Bessaïs, Eur. Phys. J. Appl. Phys. (to be published)

3. F. De Lillo, F. Cecconi, G. Lacorata, A. Vulpiani, EPL, 84 (2008)

4. L. T. De Luca, Propulsion physics (EDP Sciences, Les Ulis, 2009)

5. Rec. ITU-R SM.328-11. Recomendation ITU-R. Spectra and bandwidth of emissions. (Question ITU-R 222/1). [On-line]. Available: https://www.itu.int/dms pubrec/itur/rec/sm/R-REC-SM.328-11-200605-I!!PDF-E.pdf [April 29, 2019].

6. Dr. Kamilo Feher, Wireless Digital Communicatios: Modulation and Spread Spectrum Applications. Prentice Hall PTR (1995)

7. A. Kesteloot, C. L. Hutchinson. The ARRL Spread Spectrum Sourcebook. The American Radio Relay League, Newington, CT (1991) 